\title{
Relationship Between Atrial Oscillatory Acetylcholine Release Pattern and f-wave Frequency Modulation: a Computational and Experimental Study
}

\author{
Chiara Celotto $^{1,2}$, Carlos Sánchez ${ }^{1,2,3}$, Konstantinos A. Mountris ${ }^{1,2}$, Mostafa Abdollahpur ${ }^{4}$, Frida \\ Sandberg $^{4}$, Pablo Laguna ${ }^{1,2}$, Esther Pueyo ${ }^{1,2}$ \\ ${ }^{1}$ Aragon Institute of Engineering Research, University of Zaragoza, Spain \\ ${ }^{2}$ CIBER in Bioengineering, Biomaterials and Nanomedicine, Zaragoza, Spain \\ ${ }^{3}$ Defence University Centre, General Military Academy, Zaragoza, Spain \\ ${ }^{4}$ University of Lund, Lund, Sweden
}

\begin{abstract}
The frequency of fibrillatory waves ( $f$-waves), $F_{\mathrm{f}}$, exhibits significant variation over time, and previous studies suggest that some of this variation is related to respiratory modulation through the autonomic nervous system. In this study, we tested the hypothesis that this variation $\left(\Delta F_{\mathrm{f}}\right)$ could be related to acetylcholine concentration ([ACh]) release pattern.

Electrocardiograms were recorded from seven patients during controlled respiration before and after full vagal blockade, from which $f$-wave frequency modulation was characterized. Computational simulations in human atrial tissues were performed to assess the effects of [ACh] release pattern on $F_{\mathrm{f}}$ and compared to experimental results in humans. A cross-stimulation protocol was applied onto the tissue to initiate a rotor while cyclically varying [ACh] following a sinusoidal waveform of frequency equal to $0.125 \mathrm{~Hz}$. Different mean levels $(0.05,0.075 \mu \mathrm{M} / \mathrm{l})$ and peak-to-peak ranges $(0.1,0.05,0.025 \mu \mathrm{M} / \mathrm{l})$ of [ACh] variation were tested.

In all patients, an $f$-wave frequency modulation could be observed. In 57\% of the patients, this modulation was significantly reduced after vagal blockade. Simulations confirmed that rotor frequency variations followed the induced [ACh] patterns. Mean $F_{\mathrm{f}}$ was dependent on mean [ACh] level, while $\Delta F_{\mathrm{f}}$ was dependent on [ACh] variation range.
\end{abstract}

\section{Introduction}

The autonomic nervous system (ANS) plays a major role in the genesis and maintenance of atrial fibrillation (AF). Stimulation of the vagus nerve and, on the contrary, vagal denervation have been proven to respectively facilitate and suppress AF [1]. Furthermore, vagal stimulation, by shortening AF cycle length, has been postulated to increase the left atrial mean dominant frequency [1]. The parasympathetic neurotransmitter acetylcholine (ACh), in fact, shortens action potential (AP) duration (APD) and hyperpolarizes the resting membrane potential (RMP) in a dose dependent manner. Hyperpolarization of the RMP brings to a reduction in the maximum upstroke velocity (UV) of the AP [2], which can slow conduction velocity (CV). These effects facilitate AF by shortening the wavelength for reentry (WL), which is defined as the distance traveled by the depolarization wave during the effective refractory period (ERP) and is computed as $\mathrm{CV}$ times ERP.

In AF patients, the dominant $\mathrm{f}$-wave frequency $\left(F_{\mathrm{f}}\right)$ exhibits significant variation over time and previous studies have suggested that this variation is related to respiratory modulation through the parasympathetic nervous system $[3,4]$. In our study, we tested the hypothesis that such variation could be related to the release pattern of ACh over time.

We first analyzed clinical data from seven chronic $\mathrm{AF}$ (cAF) patients, from which electrocardiograms (ECGs) were recorded during baseline and controlled respiration, before and after full vagal blockade by atropine injection. Next, we performed in silico modeling and simulation of two-dimensional cAF human atrial tissues to assess the effects of ACh concentration ([ACh]) pattern on $F_{\mathrm{f}}$. The advantage of this in silico approach, after confirmation of agreement with clinical outcomes, is that it allows dissecting the influence of ACh release on f-wave frequency modulation independently of other factors.

\section{Methods}

\subsection{Clinical recordings}

A group of seven patients with cAF, atrioventricular block III and a permanent pacemaker were studied to investigate modulation of $F_{\mathrm{f}}$ by respiration through the parasympathetic system. The original study [4] included 
Table 1. $\overline{F_{\mathrm{f}}}$ and $\Delta F_{\mathrm{f}}$ in simulations and real cases

\begin{tabular}{|c|c|c|c|c|c|c|c|}
\hline \multicolumn{6}{|c|}{$\overline{~ S I M U L A T I O N S ~}$} & \multirow{3}{*}{\multicolumn{2}{|c|}{$\begin{array}{l}\text { EXPERIMENTS } \\
\text { Mean values } \\
\text { from patients }\end{array}$}} \\
\hline \multicolumn{2}{|c|}{ [ACh] release $0.125 \mathrm{~Hz}$} & \multicolumn{4}{|c|}{ Range (peak to peak) of [ACh] } & & \\
\hline Mean ACh & & $\mathbf{0 . 0}$ & 0.025 & 0.05 & 0.1 & & \\
\hline \multirow{3}{*}{$\mathbf{0 . 0 5} \mu \mathrm{M} / \mathbf{l}$} & $\overline{\overline{F_{\mathrm{f}}}}$ & 7.51 & 7.47 & 7.46 & 7.25 & \multirow{3}{*}{$\begin{array}{l}\overline{F_{\mathrm{f}}} \\
\sigma\end{array}$} & \multirow{3}{*}{$\begin{array}{l}6.82 \\
0.59\end{array}$} \\
\hline & $\Delta F_{\mathrm{f}}$ & 0.02 & 0.18 & 0.44 & 1.21 & & \\
\hline & $r$ & & 0.83 & 0.94 & 0.95 & & \\
\hline \multirow{3}{*}{$0.075 \mu \mathrm{M} / \mathbf{l}$} & $\overline{\overline{F_{\mathrm{f}}}}$ & 7.99 & 7.97 & 7.97 & not tested: & \multirow{3}{*}{$\begin{array}{l}\Delta F_{\mathrm{f}} \\
\sigma\end{array}$} & \multirow{3}{*}{$\begin{array}{l}0.15 \\
0.01\end{array}$} \\
\hline & $\Delta F_{\mathrm{f}}$ & 0.02 & 0.14 & 0.35 & out of & & \\
\hline & $r$ & & 0.88 & 0.95 & physiol. range & & \\
\hline
\end{tabular}

eight patients, but the f-wave signal quality was sufficient for analysis of respiratory modulation in only seven of these [5]. ECGs were recorded at rest during baseline, during $0.125 \mathrm{~Hz}$ frequency controlled respiration and during controlled respiration after full vagal blockade by atropine injection [4].

\subsection{Atrial tissue models}

Human atrial electrical activity was simulated in twodimensional 7-by-7 cm sheets of tissue. A diffusion coefficient of $0.002 \mathrm{~cm}^{2} / \mathrm{ms}$ and a transversal-to-longitudinal diffusion ratio of 0.5 were considered. This corresponds to a longitudinal conduction velocity of $44.0 \mathrm{~cm} / \mathrm{s}$ for a planar wave, in agreement with values reported for AF patients in previous studies [6].

The Courtemanche human atrial AP model was used to represent cellular electrophysiology [7]. Parasympathetic effects were incorporated into the model by including an ACh-activated potassium current, $\mathrm{I}_{\mathrm{KACh}}$, as defined in [8] with the updates proposed in [6].

Electrical remodeling associated with $\mathrm{cAF}$ was accounted for by reducing the maximal conductances of $\mathrm{I}_{\mathrm{to}}$, $\mathrm{I}_{\mathrm{CaL}}$ and $\mathrm{I}_{\mathrm{kur}}$ by $50 \%, 70 \%$ and $50 \%$, respectively, as in [9]. Regarding cAF-induced structural remodeling, various studies have reported percentages of atrial diffuse fibrosis up to $40 \%$, with a mean reported percentage of approximately $20 \%$ [10]. To simulate $20 \%$ diffuse fibrosis, $20 \%$ of nodes were randomly selected and assigned with the MacCannell model [11], an active fibroblast model that includes four membrane ionic currents. Myocytes were electrically coupled to fibroblasts with a gap junctional diffusion coefficient of $0.0005 \mathrm{~cm}^{2} / \mathrm{ms}$.

\subsection{Simulated ACh release patterns}

ACh was applied homogeneously in space while cyclically varying in time following a sinusoidal waveform of frequency equal to the $0.125 \mathrm{~Hz}$ respiratory frequency. Different mean levels $(0.05,0.075 \mu \mathrm{M} / \mathrm{l})$ and peak-to-peak variation ranges $(0.1,0.05,0.025 \mu \mathrm{M} / \mathrm{l})$ of $[\mathrm{ACh}]$ were tested, all within reported physiological ranges ( 0 - 0.1 $\mu \mathrm{M} / \mathrm{l})[12]$.

\subsection{Numerical methods and simulations}

Simulations were run using ELECTRA, an in-house software that implements the Finite Element and the Meshfree Mixed Collocation methods to solve the monodomain model. In this work, we used the Finite Element implementation. An adaptive time step ranging from 0.05 to $0.005 \mathrm{~ms}$ and a space step of $0.02 \mathrm{~cm}$ were considered. Single cells were paced at a fixed cycle length (CL) of 1000 ms for 1 minute to reach steady-state. The steady-state values of the cellular models' state variables were used for initialization of tissue models. A cross-stimulation protocol was applied onto the tissue to initiate a rotor: a first stimulus was applied at the bottom edge of the tissue and a second stimulus was applied onto a 3.5 by $3.5 \mathrm{~cm}$ square at the bottom right corner. The simulation time in atrial tissues was $10 \mathrm{~s}$. Fig. 1 illustrates voltage maps at different time instants.

\subsection{Dominant frequency characterization}

From the clinical ECGs, the f-wave signal was obtained by applying spatiotemporal QRST cancellation and $F_{\mathrm{f}}$ trend was estimated using a model-based approach. $\overline{F_{\mathrm{f}}}$ was computed as the average value of $F_{\mathrm{f}}$, while $\Delta F_{\mathrm{f}}$, the magnitude of f-wave frequency modulation, was computed as the median of the upper envelope of the bandpass filtered $F_{\mathrm{f}}$ signal [5].

From the simulations, the voltage time series from 49 evenly sampled points across the tissue (black dots in Fig.1) were analyzed. A complete 8-second respiratory period (from seconds 1 to 9) was considered. The 8 -second voltage series for one of the 49 points is illustrated in Fig. 2 (a). For each of the 49 points in the tissue, the time instant $\mathrm{t}_{\mathrm{maxUV}, \mathrm{i}}$ correspondent to the maximum UV of beat $i$ was determined (Fig. 2 (b)) and the instantaneous frequency was computed as $1 /\left(t_{\operatorname{maxUV}, i+1}-t_{\operatorname{maxUV}, i}\right)$ (Fig. 2 (c)), for all beat indices $i$ in the recording. Next, averaging (in 




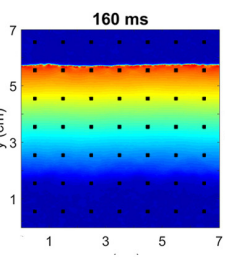

$270 \mathrm{~ms}$





$300 \mathrm{~ms}$

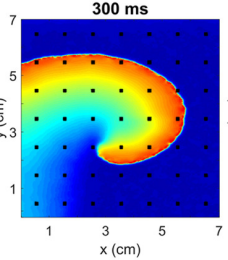

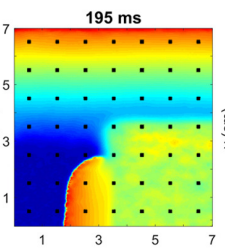

$330 \mathrm{~ms}$

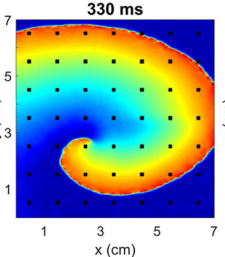

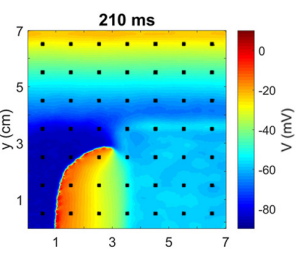

$390 \mathrm{~ms}$

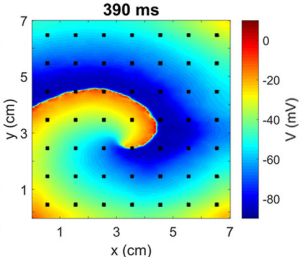

Figure 1. Voltage maps at different time instants for [ACh] varying around $0.05 \mu \mathrm{M} / 1$ with peak-to-peak range of $0.1 \mu \mathrm{M} / 1$. At $180 \mathrm{~ms}$ the second stimulus applied onto the bottom-right corner of the tissue is visible. The 49 black dots in each snapshot represent the points used in the computation of $F_{\mathrm{f}}$.
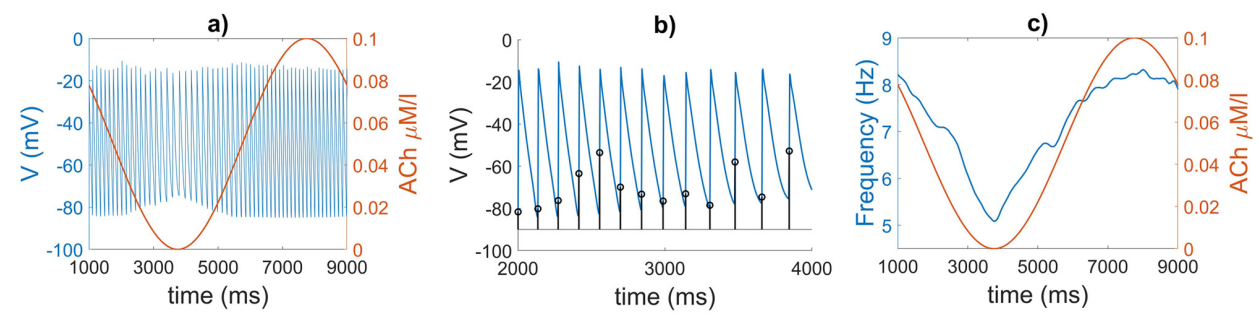

d)
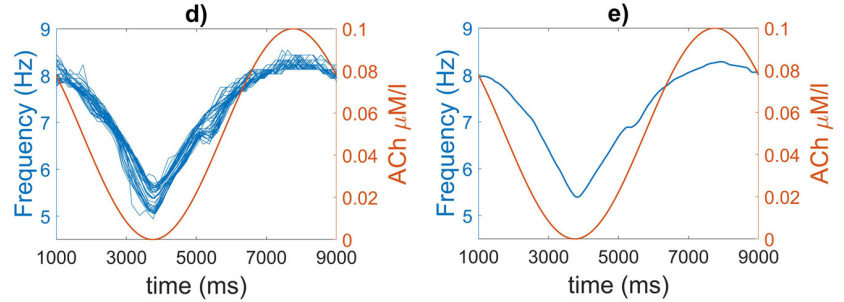

Figure 2. a) Voltage time series for one point in the tissue (point 12 out of the 49 points in Fig. 1 starting from bottom left and moving right/up) (blue line) in relation to [ACh] temporal variation (red line). b) Identification of the time instants of maximum AP upstroke velocity for the same individual spatial point. c) Instantaneous frequency for the same individual spatial point (blue line) in relation to [ACh] variation (red line). d) Instantaneous frequencies computed for all spatial points in the tissue after removal of those correspondent to the rotor core. e) Time series of the dominant frequency for the tissue, $F_{\mathrm{f}}$, obtained by spatial trimmed averaging (blue line) in relation to $[\mathrm{ACh}]$ variation (red line).

space) was performed to compute the tissue dominant frequency $F_{\mathrm{f}}$ along time. Specifically, $F_{\mathrm{f}}$ was computed as a trimmed mean of the instantaneous frequencies over the 49 points, discarding the $5 \%$ extremes to exclude the chaotic signals typically corresponding to the rotor core (Fig. 2 (d) and (e)). $\overline{F_{\mathrm{f}}}$ and $\Delta F_{\mathrm{f}}$ were obtained from the estimated $F_{\mathrm{f}}$ time series using the same method as for the clinical signals.

\section{Results}

In all patients' ECGs, an f-wave frequency modulation was observed. In $57 \%$ of the patients, this modulation was significantly reduced after the injection of atropine. Mean results over patients in terms of mean dominant frequency $\overline{F_{\mathrm{f}}}$, magnitude of modulation $\Delta F_{\mathrm{f}}$ and standard deviation $\sigma$ are reported in Table 1. Simulation results confirmed that the rotor frequency variations followed the induced [ACh] patterns (Fig. 3), with correlation coefficient $r$ above 0.83 in all cases, as reported in Table 1. Furthermore, $r$ increases with the mean level and the variation range of $[\mathrm{ACh}]$ since the dominance of the phenomenon increases with increasing [ACh]. The mean dominant frequency $\overline{F_{\mathrm{f}}}$ was found to be dependent on the mean [ACh] level, while its peak-to-peak variation $\Delta F_{\mathrm{f}}$ was dependent 
on the $[\mathrm{ACh}]$ variation range, with increases in mean and range of [ACh] leading to increases in $\overline{F_{\mathrm{f}}}$ and $\Delta F_{\mathrm{f}}$, respectively. An [ACh] temporal variation of $0.05 \mu \mathrm{M} / 1$ mean value and $0.025 \mu \mathrm{M} / 1$ peak-to-peak range equal was the one providing results closer to those measured from the patients.
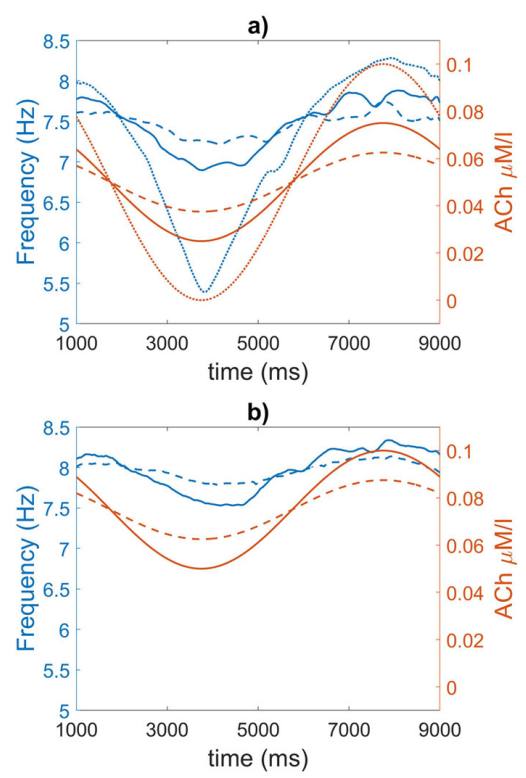

Figure 3. $\quad F_{\mathrm{f}}$ (blue) and [ACh] (red) for mean [ACh] of $0.05 \mu \mathrm{M} / 1$ (a) and $0.075 \mu \mathrm{M} / 1$ (b). Dashed/solid/dotted lines represent $0.025 / 0.05 / 0.1 \mu \mathrm{M} / 1 \quad$ [ACh] variation ranges. In panel (b) the maximum [ACh] variation range of $0.1 \mu \mathrm{M} / 1$ is not represented since it is out of the physiological range.

\section{Conclusions}

The pattern of [ACh] release could be an important factor involved in f-wave frequency modulation. Further studies will help to elucidate the contribution of other factors and to ascertain whether [ACh] variations could be monitored from f-wave analysis.

\section{Acknowledgments}

This work was supported by the European Research Council through grant ERC-StG 638284, EU H2020 Marie Skłodowska-Curie ITN grant No.766082 MY-ATRIA, by MICINN (Spain) through projects PID2019-105674RBI00 and PID2019-104881RB-I00 and by Gobierno de Aragón through project LMP124-18 and Reference Group BSICoS T39-20R cofunded by FEDER 2014-2020. Computations were performed by ICTS NANBIOSIS (HPC Unit at University of Zaragoza).

\section{References}

[1] Chang HY, Lo LW, Chou YH, Lin WL, Lin YJ, Yamada $\mathrm{S}$, Chen SA. Effect of vagotomy on the activity of cardiac autonomic ganglia: Insight from left atrial high density frequency mapping. International Journal of Cardiology 2016; 220:435-439.

[2] Rohr S, Kucera JP, Kleber AG. Slow conduction in cardiac tissue, i: Effects of a reduction of excitability versus a reduction of electrical coupling on microconduction. Circulation Research Oct. 1998;83(8):781-794.

[3] Stridh M, Meurling C, Olsson B, Sörnmo L. Detection of autonomic modulation in permanent atrial fibrillation. Med Biol Eng Comput Nov. 2003;41(6):625-629.

[4] Holmqvist F, Stridh M, Waktare JEP, Brandt J, Sörnmo L, Roijer A, Meurling CJ. Rapid fluctuations in atrial fibrillatory electrophysiology detected during controlled respiration. American Journal of Physiology Heart and Circulatory Physiology Aug. 2005;289(2):H754-H760.

[5] Abdollahpur M, Holmqvist F, Platonov PG, Sandberg F. Respiratory modulation in permanent atrial fibrillation. Computing in Cardiology CinC Rimini Sept. 2020;

[6] Bayer JD, Boukens BJ, Krul SPJ, Roney CH, Driessen AHG, Berger WR, van den Berg NWE, Verkerk AO, Vigmond EJ, Coronel R, de Groot JR. Acetylcholine delays atrial activation to facilitate atrial fibrillation. Front Physiol Sept. 2019;10:1105.

[7] Courtemanche M, Ramirez RJ, Nattel S. Ionic mechanisms underlying human atrial action potential properties: insights from a mathematical model. American Journal of Physiology Heart and Circulatory Physiology Jul. 1998; 275(1):H301-H321.

[8] Kneller J, Zou R, Vigmond EJ, Wang Z, Leon LJ, Nattel S. Cholinergic atrial fibrillation in a computer model of a twodimensional sheet of canine atrial cells with realistic ionic properties. Circ Res May 2002;90(9).

[9] Courtemanche M. Ionic targets for drug therapy and atrial fibrillation-induced electrical remodeling: insights from a mathematical model. Cardiovascular Research May 1999; 42(2):477-489.

[10] McDowell KS, Zahid S, Vadakkumpadan F, Blauer J, MacLeod RS, Trayanova NA. Virtual electrophysiological study of atrial fibrillation in fibrotic remodeling. PLoS ONE Feb. 2015;10(2):e0117110.

[11] Andrew MacCannell K, Bazzazi H, Chilton L, Shibukawa Y, Clark RB, Giles WR. A mathematical model of electrotonic interactions between ventricular myocytes and fibroblasts. Biophysical Journal June 2007;92(11):4121-4132.

[12] Song Y, Shryock JC, Belardinelli L. Potentiating effect of acetylcholine on stimulation by isoproterenol of Ltype $\mathrm{Ca} 2+$ current and arrhythmogenic triggered activity in guinea pig ventricular myocytes. J Cardiovasc Electrophysiol 1998;9(7):718-726.

Address for correspondence:

Chiara Celotto

Universidad de Zaragoza, Campus Río Ebro, Edif.I+D, C/ Poeta Mariano Esquillor, s/n, 50018 Zaragoza

chiaracelotto@unizar.es 